\title{
BC-819 Plasmid/Polyethylenimine Complex
}

National Cancer Institute

\section{Source}

National Cancer Institute. BC-819 Plasmid/Polyethylenimine Complex. NCI Thesaurus.

Code C107685.

A plasmid DNA encoding for the A fragment of Diphtheria Toxin (DTA) under the control of the $\mathrm{H} 19$ gene promoter (BC-819 or DTA-H19) and mixed with the transfectant polyethylenimine (PEI), with potential antineoplastic activity. Upon administration, the PEI moiety enhances the entry of the agent into rapidly dividing cells. Upon cell entry, activation of the $\mathrm{H} 19$ gene promoter-containing plasmids and DTA expression are limited to tumor cells, as high levels of H19 expression are only found in tumor cells. DTA disrupts protein synthesis. Tumor-cell selective expression of this toxin leads to the selective destruction of the tumor while sparing healthy, normal cells. H19, an oncofetal, regulatory RNA, is overexpressed in certain cancer cells while its expression in normal cells is minimal or absent; it plays a key role in cancer progression, angiogenesis and metastasis. 